\title{
Spontaneous coordinated activity in cultured networks: analysis of multiple ignition sites, primary circuits, and burst phase delay distributions
}

\author{
Michael I. Ham ${ }^{1,2}$,Luís M. A. Bettencourt ${ }^{2,3}$,Floyd D. McDaniel ${ }^{1}$,Guenter W. Gross ${ }^{4}$ \\ ${ }^{1}$ Department of Physics \\ PO Box 311427, Univeristy of North Texas, \\ Denton Texas 76203 USA \\ ${ }^{2} T-7$ and CNLS, Theoretical Division \\ MS B284 Los Alamos National Laboratory, \\ Los Alamos NM 87545 \\ ${ }^{3}$ Santa Fe Institute \\ 1399 Hyde Park Road, Santa Fe NM 87501, USA \\ ${ }^{4}$ Center for Network Neuroscience \\ University of North Texas, Denton TX 76203, USA
}

(Dated: February 12, 2008)

\begin{abstract}
All higher order central nervous systems exhibit spontaneous neural activity, though the purpose and mechanistic origin of such activity remains poorly understood. We quantitatively analyzed the ignition and spread of collective spontaneous electrophysiological activity in networks of cultured cortical neurons growing on microelectrode arrays. Leader neurons, which form a mono-synaptically connected primary circuit, and initiate a majority of network bursts were found to be a small subset of recorded neurons. Leader/follower firing delay times formed temporally stable positively skewed distributions. Blocking inhibitory synapses usually resulted in shorter delay times with reduced variance. These distributions are characterizations of general aspects of internal network dynamics and provide estimates of pair-wise synaptic distances. The resulting analysis produced specific quantitative constraints and insights into the activation patterns of collective neuronal activity in self-organized cortical networks, which may prove useful for models emulating spontaneously active systems.
\end{abstract}

\section{INTRODUCTION}

No neural system is ever completely absent of electrophysiological activity. The putative roles of such spontaneous dynamics, although still relatively poorly understood, range from synaptic development and maintenance $[3,21,26,31,34]$ to anticipatory states (e.g. [35]) that help animals reach rapid decisions from limited sensory input. Understanding spontaneous activity and the patterns of interaction between neurons in circuits are, therefore, issues of substantial importance. A large body of theoretical analysis and experimental data indicates that cortical neuronal networks growing on microelectrode arrays (MEAs) in vitro provide useful experimental models of neural assembly (e.g. $[4,9,33])$ despite obvious limitations inherent to extrapolations from in vitro to in vivo systems (see for e.g. [6, 25]). In this manuscript we perform an extensive quantitative analysis of patterns of network burst initiation in cortical neural networks in vitro. These spontaneous collective high frequency action potential discharges are major features of such systems $[2,8,10,18,19,23,24,36-38]$ and can influence learning and information processing by changing synaptic properties $[1,5]$. Previous theoretical and experimental research showed that multiple ignition sites, sometimes termed initiation loci [24], privileged neurons [11], and even initiation zones [12], create network bursts by recruiting constituent neurons. However, to date, very little has been done towards quantifying network initiation patterns resulting from such burst leader sites. Here, temporal relationships between leader (first spike in the network burst) and follower neurons were used to reconstruct network relationships amongst recorded neurons using a first-spike-in-burst analysis method. This methodology also identified changes in network ignition site statistics after disinhibition with the GABAA blocker bicuculline. Extrapolation of these distributions produced minimum response delays, which were then used to estimate the distance, in terms of number of synapses, between neurons. This approach provides new statistical views of functional connectivity between neurons and several general quantitative characterizations of the internal dynamics of living cortical networks that should be reproduced in models of small to medium sized neural networks.

\section{METHODS}

\section{Microelectrode Array (MEA) Fabrication}

In-house MEA fabrication is described in previous publications [15, 17]. Briefly, commercially available glass plates with a 100-nanometer layer of indium-tin-oxide (ITO, Applied Films Corp., Boulder, CO) were photo etched to create a recording matrix of 64 electrodes mea- 


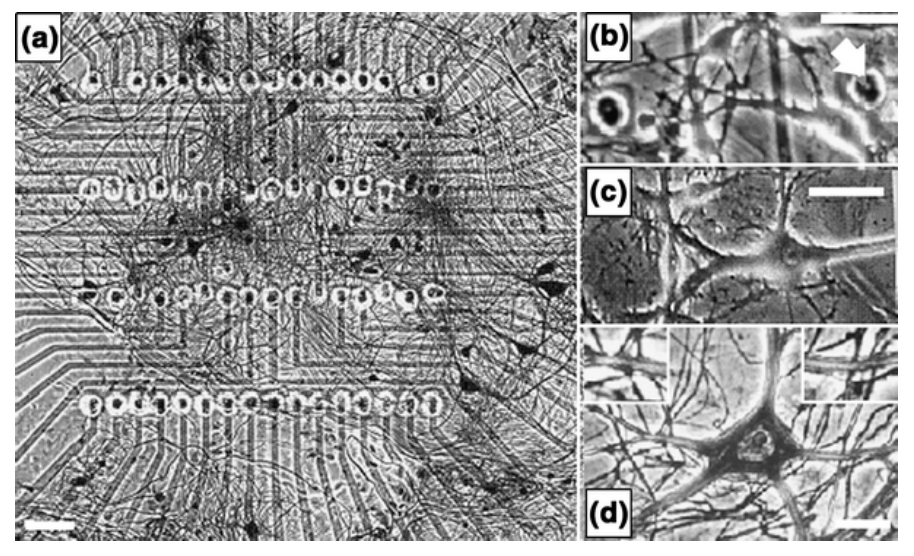

FIG. 1: Neuronal networks in cell culture. (A) Network on a 64-electrode recording matrix. The transparent indium-tin oxide conductors are $8 \mu \mathrm{m}$ wide and 1000Åthick. (92 days in vitro; Bodian stain Bars: $40 \mu \mathrm{m}$ ). (B-C) Living neurons on MEAs (phase contrast; bars: $40 \mu \mathrm{m}$ ). Gold-plated exposed ITO conductors are shown by arrows in (B). Transparent indium-tin oxide (ITO) conductors allow extensive optical access to the network morphology. (D) Single neuron showing synaptic profiles, enlarged in inserts (bar: $20 \mu \mathrm{m}$, neurofilament antibody stain)

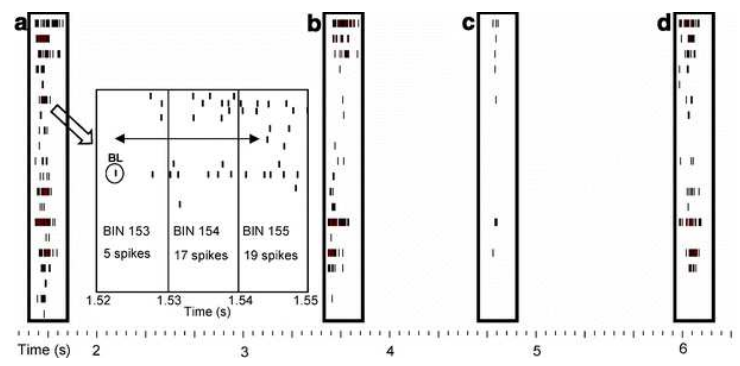

FIG. 2: Raster displays of 26 frontal cortex units participating in coordinated bursting. Three network bursts (Boxes 1, 2, 4) and one aborted network burst (Box 3) occur during the first 6 seconds of recorded activity. Inset depicts the first 30 ms of network burst 1 . The burst leader (BL) and a response delay (double headed arrow) are depicted. The bin number and spikes per bin are indicated.

suring 8-10 $\mathrm{m}$ in width and conductors leading to peripheral amplifier contacts on a $5 \times 5 \mathrm{~cm}$ glass plate. These plates were then spin-insulated with methyltrimethoxysilane resin, cured, and de-insulated at the electrode tips with single laser shots. Exposed electrode terminals were electroplated with colloidal gold to decrease impedance at $1 \mathrm{kHz}$ to approximately $0.8 \mathrm{M}$ ?. To improve cell adhesion the matrix region and surrounding area $(3 \mathrm{~mm}$ in diameter) were butane flamed followed by application of poly-D-lysine and laminin. These microelectrode arrays, featuring substrate integrated thin film conductors allow long-term, extracellular microvolt recording of action potentials from 64 discrete sites in a neuronal network.

\section{Cell Culture}

Frontal cortices were dissected from 16 to 17 day old mouse embryos. The tissue was mechanically minced, enzymatically dissociated, triturated, and combined with medium (Dulbeccos Modified Eagles Medium (DMEM) supplemented with $10 \%$ fetal bovine serum and $10 \%$ horse serum). Dissociated cells (100k / ml) were seeded on MEAs with medium addition after cells had adhered (usually 2-3 hrs). After 5 days, cultures were fed DMEM supplemented with $5 \%$ horse serum (DMEM-5). Greater detail is provided in earlier publications [14]. Cultures were incubated at $37 \mathrm{C}$ in a $10 \% \mathrm{CO} 2$ atmosphere with $50 \%$ medium changes performed twice a week until used for experiments. Cultures matured for a minimum of three weeks to allow activity patterns to become stable $[19,20,30]$ and useful for quantitative analyses [32]. Fig $1 \mathrm{~A}$ depicts a mature culture on an MEA matrix, with B-D showing constituent neurons both living and after neurofilament antibody staining.

\section{Electrophysiological Data Acquisition}

For electrophysiological recordings, cultures were assembled into a recording apparatus on an inverted microscope connected to a two-stage, 64 channel amplification and signal processing system (Plexon Inc., Dallas). Cultures were maintained at a temperature of $37 \mathrm{C}$, and $\mathrm{pH}$ of 7.4. The $\mathrm{pH}$ was maintained by a $10 \mathrm{ml} / \mathrm{min}$ flow of $10 \% \mathrm{CO} 2$ in air into a cap on the chamber block featuring a heated ITO window to prevent condensation. Water evaporation was compensated by a syringe pump (Harvard Instruments) with the addition of 60 to $70 \mu \mathrm{l} /$ hr sterile water. Details of chamber assembly and recording procedures can be found in previous papers [14, 15]. Total system gain was set at $10 \mathrm{k}$ and action potential (AP) activity with a sampling resolution of $25 \mu$ s was recorded for later analysis.

\section{Network Burst and Burst Leader Identification}

To identify network bursts from data, recordings were first partitioned into $10 \mathrm{~ms}$ bins and the total number of spikes per bin was determined (Fig 2C). An upper and lower threshold algorithm was used to identify network bursts. The upper threshold was selected by visual inspection of spike patterns, using the Neural Explorer 1-D viewer (Nex Technologies, Littleton, MA), such that normal background activity would not reach this level. Networks with many constituents generally needed higher upper thresholds. The lower threshold was set to an activity level just above the normal non-bursting background spike activity. Since frontal cortex cultures show 


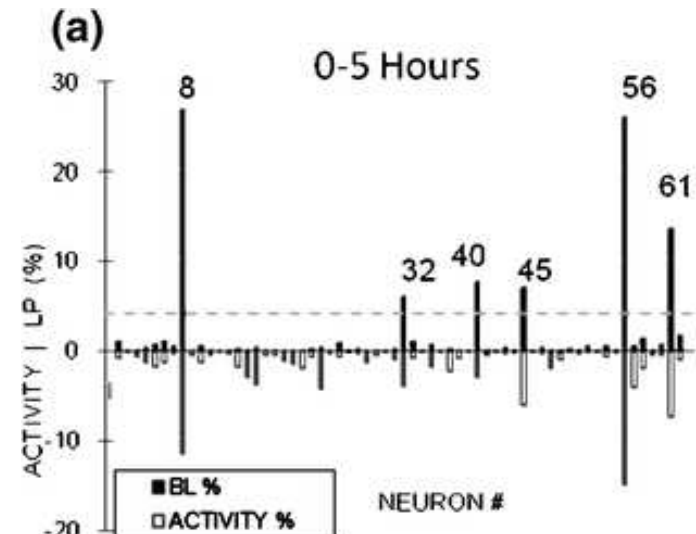

(b)

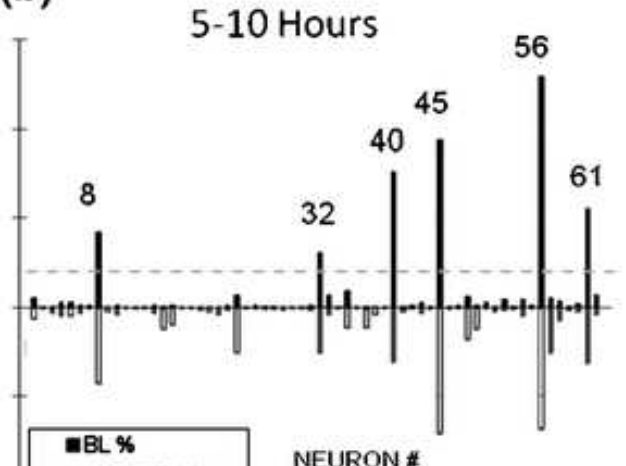

(c)

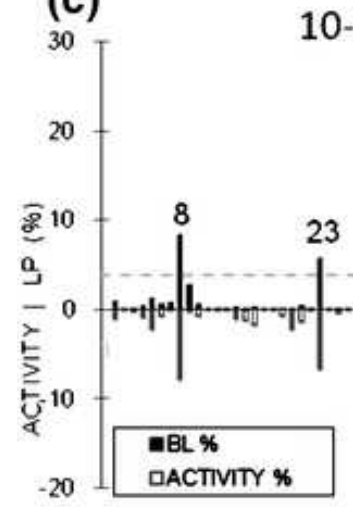

10-15 Hours

(d)

口ACTIVITY \%

NEURON \#

\section{(d) 15 - 20 Hours (bicuculline)}

(e)

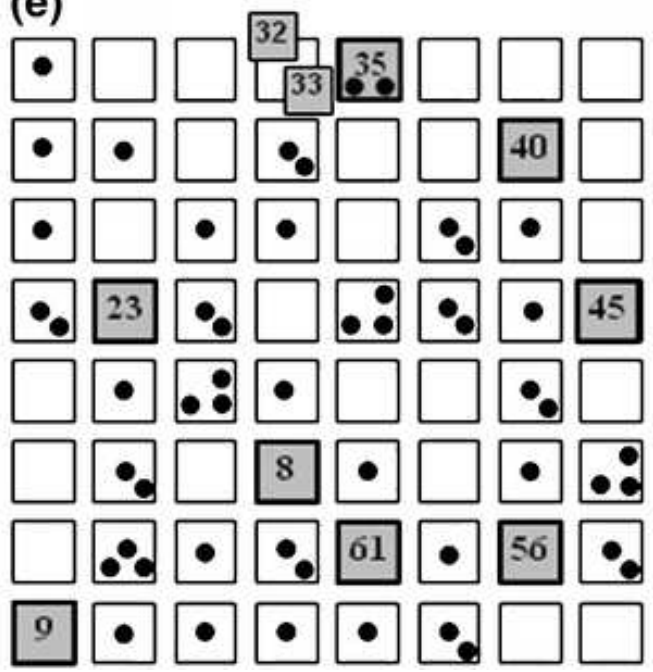

FIG. 3: Burst leader probability (LP, positive) and percent spike activity (negative) for four consecutive 5 hr time periods (A-D). Dashed lines show selected major burst leadership thresholds. During native spontaneous activity (A-C), the pool of burst leaders remains almost constant, although the LP of individual units changes. (D) Pharmacological manipulation with 40 $\mu$ of bicuculline causes changes in the pool of burst leaders. (E) Location of burst leaders and followers on the $8 \mathrm{x} 8$ recording matrix (electrodes are separated by $150 \mu$ ). Numbers represent major burst leaders and dots show followers. Multiple dots and/or numbers per electrode represent the number of units monitored at that site.

very little activity between network bursts (Fig $2 \mathrm{~A})$, the lower threshold was often set to 1 or 2 spikes.

The algorithm seeks the first bin with a number of spikes at least equal to the lower threshold. Once found, two possible scenarios were examined: (1) the bin contains a number of spikes greater than or equal to the upper threshold. This indicates the start of a global burst. The burst continues as long as consecutive bins contain a 


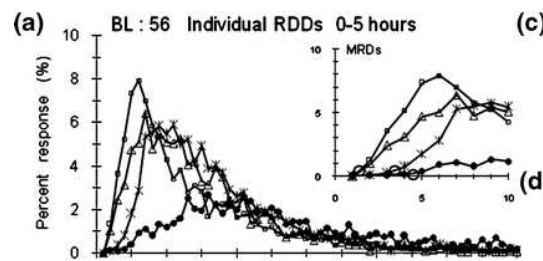

(b)
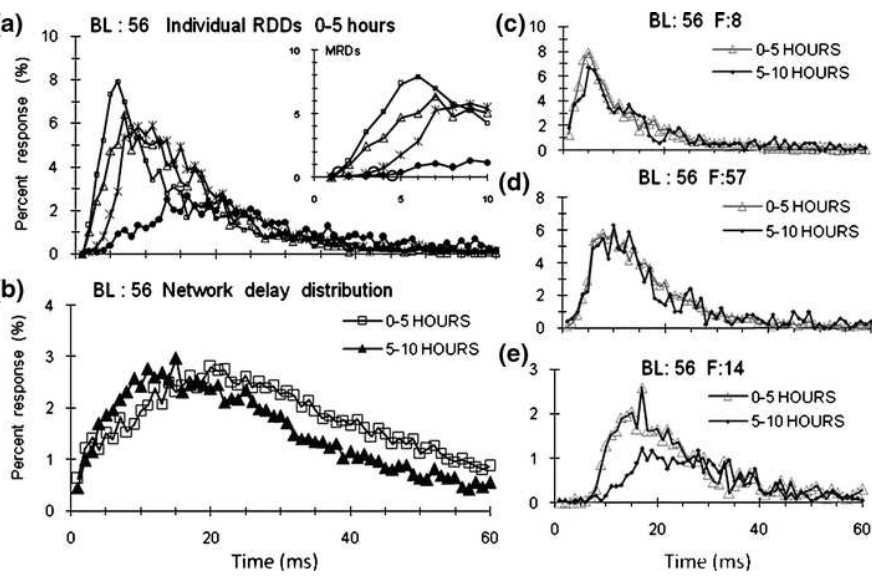

FIG. 4: Examples of burst response delay distributions generated by the same burst leader. (A) RDDs of four different followers to BL 56. Each shows unique MRDs (inset, circles) and peak delays. (B) Network phase delay distributions (summation of all RDDs to BL 56) during two five hour periods. (C-E) Stability of individual RDDs during two consecutive five hour periods. MRDs and shape remain similar. Data from network 1 .

number of spikes that satisfy the lower threshold. (2) The upper threshold is not reached. The bin is marked as the Potential Beginning Of Burst (P-BOB). The algorithm examines consecutive $10 \mathrm{~ms}$ bins searching for one that satisfies the upper threshold, or one that falls below the lower threshold. If a consecutive bin reaches or exceeds the upper threshold therefore becoming a network burst, it is identified as starting at the P-BOB. However, if a consecutive bin falls below the lower threshold without reaching the upper threshold no global burst is logged. All network bursts end when a consecutive bin falls below the lower threshold. However, network bursts with activity gaps of less than $100 \mathrm{~ms}$ were combined. Once all network bursts were identified, the first timestamp and associated neuron of each global burst were recorded. This neuron was denoted the burst leader (BL, Fig. 2C). Additionally, the first spikes of all followers were logged to determine response delays (phase delays). Over a long enough period of time (usually 5 hours), a statistical picture of burst leader probability emerges.

\section{Major burst leaders, response delay distributions and network delay distributions}

For detailed statistical analysis we define major burst leaders (MBLs) as neurons that lead at least 4\% (arbitrarily set) of all network bursts (Fig 3). We define a response delay as the time between MBL onset and a followers first spike. To be included in our statistical treatment, response delays must occur within $100 \mathrm{~ms}$, which is commensurate with the duration of the network
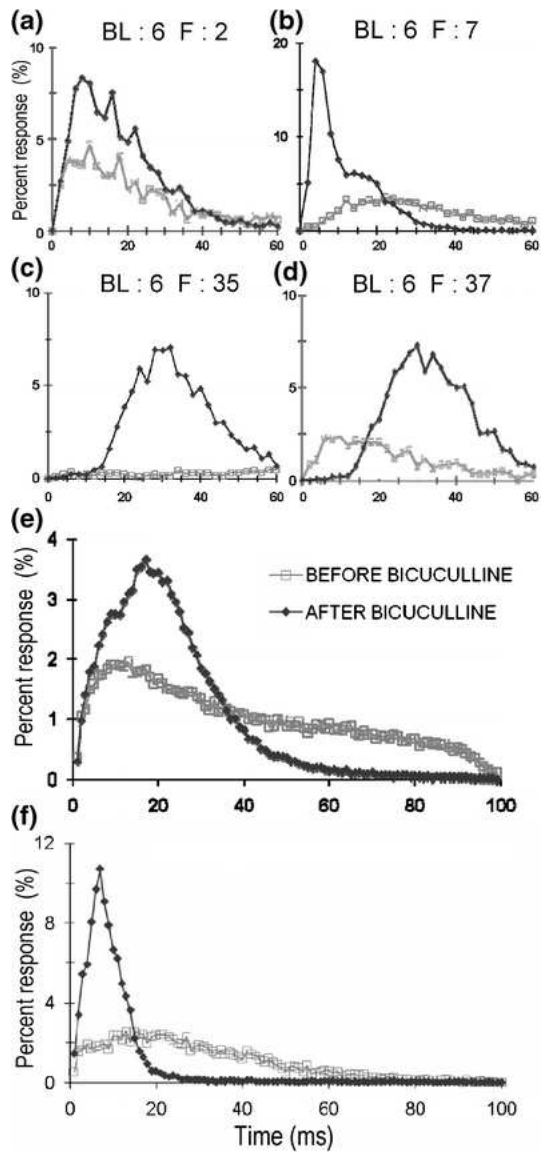

FIG. 5: Response delay distributions before and after the addition of bicuculline. Colored shapes represent distributions after bicuculline has been added. (A) Follower 2 increases its responsiveness to $\mathrm{BL} 6$, but maintains overall distribution shape. (B) Bicuculline makes follower 7 respond faster and with greater precision to burst leader 6. (C) Conversely, follower 37 responds later to BL 6. (D) Addition of bicuculline made neuron 35 responsive to the burst leader. (E) Network delay distribution (NDD) showing followers of burst leader 6 responding faster and with less variability after bicuculline. (F) NDD highlighting extensive distribution shift to shorter delays in response to bicuculline. A-E : Network 2; F : Network 3

burst initiation period [11]. Response delay distributions (RDDs) were created for MBL/follower pairs via normalized (by number of network bursts associated with the MBL) frequency histograms, in $1 \mathrm{~ms}$ time bins, over ensembles of burst events. When MBLs did not lead they were considered followers. Combining all RDDs for different followers with the same MBL and normalizing the resulting distribution generates an MBL's network delay distribution. We analyze three different features of RDDs: 1) Peak delay: the delay time corresponding with the peak of the distribution. 2) Paired response correlations (PRCs): which measure the follower's participation (range $0-100 \%$ ) in network bursts led by the MBL. 3) 

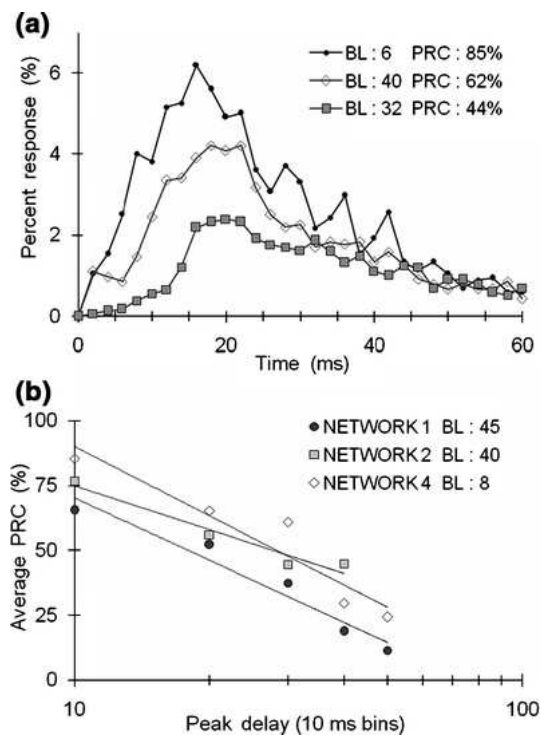

FIG. 6: (A) RDDs of a single follower to three different burst leaders demonstrating unique influences of burst leaders on a single follower. In addition such pairs also have unique response correlations (PRCs) (B) PRC as a function of peak delays (10 ms bins) showing that strong correlations are associated with short peak delays. Logarithmic trend lines.

Minimum response delays (MRDs): which is the shortest time delay corresponding to $10 \%$ of the peak response frequency and represents the fastest time in which a signal can pass between the MBL and follower. MRDs of about $2 \mathrm{~ms}$ correlate to the minimum signal delay between mono-synaptically connected neuronal pairs ([27]; minimum delay $2 \mathrm{~ms}$, avg delay $4 \mathrm{~ms}$ ).

The data set for the reported results consists of ten frontal cortex cultures, eight of which were subjected to disinhibition with $40 \mathrm{M}$ of bicuculline after at least five hours of normal (native) activity (summarized in Table 1). Bicuculline is an antagonist of the GABAA receptor that blocks the hyperpolarization effect of GABA in a competitive manner [7] When applied to frontal cortex cultures in saturating concentrations (40-60 M), it causes intensification and greater coordination of bursting with increased burst durations and average number of spikes in bursts $[22,29]$.

\section{RESULTS}

\section{Burst Leader Characteristics}

The 10 networks studied averaged about 1000 network bursts per hour. For an average of 42 discriminated units per culture (Table 1) this generates approximately 42,000 individual bursts/hour for analysis. Nearly all recorded neurons lead at least one network burst per hour, but only a small subset of recorded neurons (average 17\%; range 11-35\%) were major burst leaders (MBLs, Table 1). Individually identified MBLs most likely belong to a small cluster of neurons, as suggested by Gross and Kowalski [16] and shown recently in unidimensional networks by Feinerman et al. [12]. MBLs statistically led a similar amount of network bursts in normal medium (DMEM-5) and in medium containing the GABA channel blocker bicuculline (Table 1).

The set of MBLs remained approximately constant under native spontaneous activity conditions. Fig 3A$\mathrm{C}$ shows native medium burst leadership statistics from network 1 during three consecutive five-hour recordings. Six neurons were MBLs during the first 10 hours (A\&B), seven (previous six included) were major leaders during the third $5 \mathrm{hr}$ period $(\mathrm{C})$, and six were identified after application of bicuculline (D). Under bicuculline, only three previous leaders, units 8,32 and 56 remained, while three new leaders 9,33 and 35 emerged. Note that identification numbers do not represent positions in the recording matrix. Actual leader locations on the matrix are shown in Fig $3 \mathrm{E}$, which depicts an $8 \times 8$ microelectrode matrix with vertical and horizontal electrode separation of 150 $\mu \mathrm{m}$. Shaded boxes represent MBLs with numbers corresponding to those in A-D. Followers are depicted by dots. It can be seen that the burst leader sites were distributed spatially over the entire matrix.

Burst leadership is not a direct function of neuronal spike rates. In Figs 3A-D, percent spike activity (number of individual spikes / total number of network spikes) is compared to burst leadership. During native activity, all MBLs had leadership percentages (positive bars) greater than percent spike activity (negative bars). Conversely many active spiking units showed no burst leadership beyond $1 \%$, which we consider to be in the noise level. While MBLs typically exhibited a high percent spike activity, high activity did not ensure that a neuron would be an MBL.

\section{Leader/follower pair-based response delays and distributions}

Network bursts are variable events in terms of the detailed firing patterns between neurons (Fig 2). Therefore, relationships between network neurons are of a statistical nature. Typically, cortical network relationships are established by comparing the total spike output pattern of one neuron with the total spike output pattern of another neuron or group of neurons. (e.g. [2, 4, 28]). Here we take a different approach and only use the first spike each participating neuron makes in each network burst. Fig 4A shows four different response delay distributions (RDDs; Methods), which have the same burst leader but different followers. The distribution peak delay (most probable delay) represents the sum of three primary delay mechanisms: conduction, synaptic, and processing. 
(a)

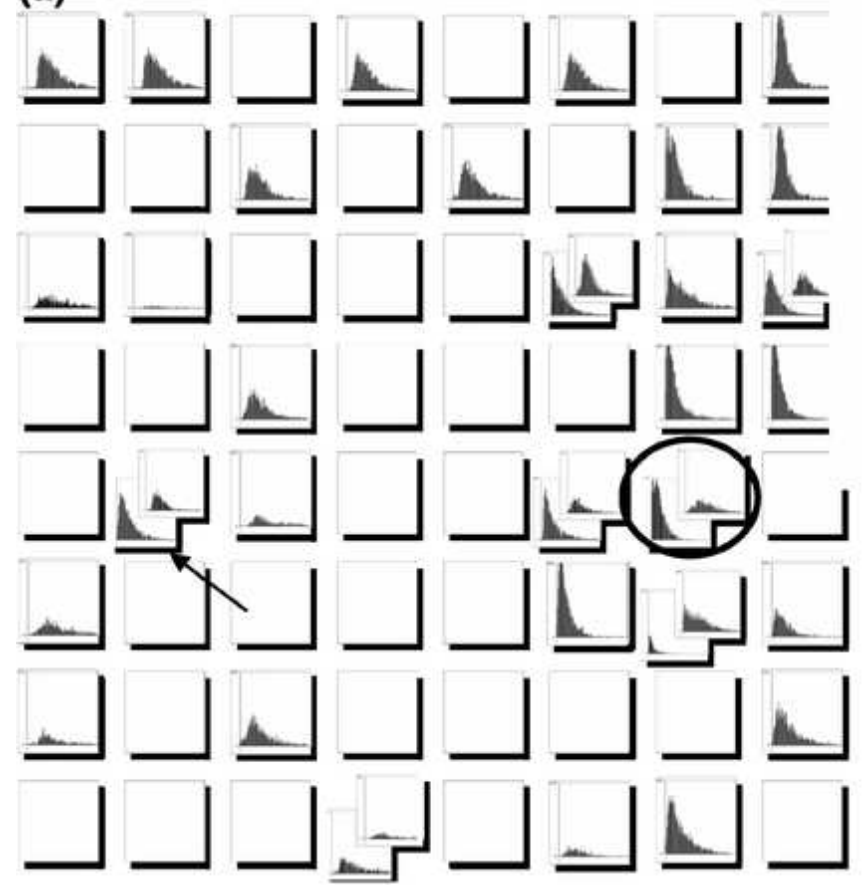

(b)

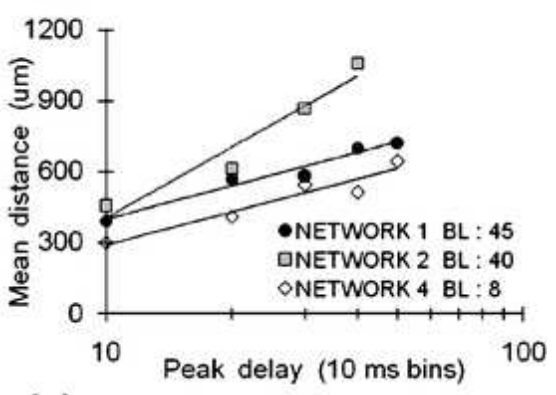

(c)

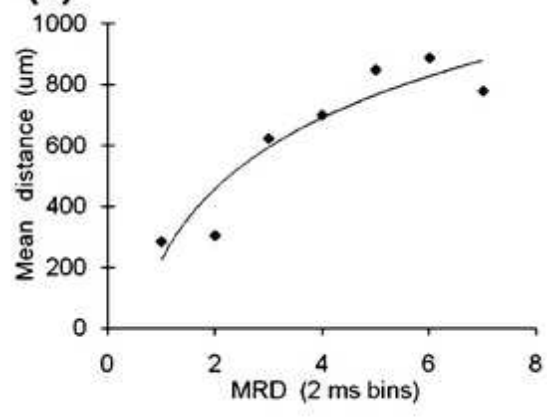

FIG. 7: (A) RDDs for a burst leader (circled) are shown in their recording matrix location. It should be noted that the two distributions within the circle are follower neurons recorded on the same electrode. Multiple RDDs are depicted for electrodes with multiple units. (B) RDD peak delays in 10ms bins, plotted against the average distance from the burst leader. (C) Relationship between part A's MRDs (2 ms bins) and average distance. Logarithmic trend lines.

\begin{tabular}{|c|c|c|c|c|c|c|}
\hline \multirow[b]{2}{*}{ N\# } & \multirow[b]{2}{*}{ U\# } & \multirow[b]{2}{*}{ Age } & \multicolumn{2}{|c|}{ DMEM-5 } & \multicolumn{2}{|c|}{$+40 \mu \mathrm{M}$ Bicuculline } \\
\hline & & & MBL & NBM & $\| \mathrm{M} \% \mathrm{U}$ & NBMBL \\
\hline 1 & 62 & 41 & 11 & 75 & 10 & 81 \\
\hline 2 & 41 & 44 & 24 & 81 & 17 & 66 \\
\hline 3 & 20 & 33 & 35 & 92 & 15 & 81 \\
\hline 4 & 33 & 51 & 12 & 67 & 21 & 82 \\
\hline 5 & 50 & 41 & 12 & 94 & 12 & 78 \\
\hline 6 & 37 & 39 & 14 & 90 & 14 & 82 \\
\hline 7 & 34 & 25 & 24 & 76 & 15 & 85 \\
\hline 8 & 42 & 52 & 14 & 83 & 19 & 89 \\
\hline 9 & 21 & 125 & 10 & 97 & $\mathrm{~N} / \mathrm{A} \mathrm{N} / \mathrm{A}$ & \\
\hline 10 & 48 & 42 & 10 & 83 & $\mathrm{~N} / \mathrm{A}$ & $\mathrm{N} / \mathrm{A}$ \\
\hline Means & 42 & 48 & $\pm 3(\mathrm{SD})$ & $84 \pm 9.5$ & $15 \pm 3.6$ & $81 \pm 6.7$ \\
\hline
\end{tabular}

TABLE I: N\#: Network Number; U\#: Number of units; NBM: Number of network bursts led by MBLs (\%); M\%U: MBLs as a percent of all units; NBMBL: Number of network bursts led by MBLs (\%)

It is expected that the latter is highly variable and computational in nature as multiple excitatory and inhibitory synaptic inputs create a complex array of morphological and physiological factors that influence the spike outputs of specific neurons. To these factors must be added inherent cellular properties such as threshold and basic firing behavior. Such complexity is reflected in the shape of an MBL/follower RDD. Minimum response delay (MRD; Methods) values (Fig 4A), which are expected to have minimal processing delay, were approximately $2 \mathrm{~ms}$ for most followers, but ranged up to $20 \mathrm{~ms}$. In Fig $4 \mathrm{E}$ an MRD of $8 \mathrm{~ms}$ can be seen.

RDDs remained relatively stable in consecutive five hour recordings (Figs 4C-D). Fig 4B demonstrates the stability of the network delay distribution using MBL 56 as an example. Temporal firing stability has been shown previously between recorded neurons when a neurons total spike output was compared to the total spike output of another neuron [28], The stability of pair-wise relationships, whether from first spike in burst, or all spikes, could indicate that the same circuit used to initiate network bursts is also used to maintain activity.

\section{Blockage of inhibitory synapses: Effects on phase delays and MBL pools}

Loss of inhibition, induced by the application of $40 \mathrm{M}$ bicuculline, changes the makeup of the MBL group. In networks 1-8, there were fifty-two MBLs during native activity and forty-eight after bicuculline was added. Only twenty-five of the latter were present during native activity while the remaining twenty-three were new MBLs. In all networks, bicuculline also changed the form of the re- 
sponse delay relationships between neural pairs. Figs 5AD show four examples of the observed effects bicuculline had on follower neuron responsiveness. (1) Increased responsiveness without major changes in distribution shape (5A); (2) shifting of distributions to shorter phase delays (5B), (3) distribution peak and the MRD shift to higher phase delays (5C) and (4) participation by previously unresponsive neurons (5D). Overall, $51 \%$ of the followers had MRDs shift to shorter phase delays, $18.5 \%$ shifted to longer peak delays and $30 \%$ showed no MRD change. There were no observed cases where the addition of bicuculline resulted in a follower becoming less responsive to a burst leader.

Such results show that studies of neural relationships and connectivity can be enhanced by specific pharmacological manipulations. An example is shown in Fig $5 \mathrm{D}$ where neuron 35 is unresponsive to MBL 6 in native medium. However, the blockage of GABAA receptors immediately triggered strong pair-wise responses. This indicates that, in the native state, inhibitory influences between 6 and 35 dominated, but excitatory connections existed. In future experiments, pharmacological control over synaptic driving forces will become increasingly important in the quest for determination of functional connectivity.

Since each of the four bicuculline induced RDD changes can happen to followers of the same burst leader, observing the overall population effect requires examining the network delay distributions. In the network delay distribution shown in Fig 5E, the bulk of network responses occurred earlier despite the peak delay increase. In this case, all response delays occurred within $70 \mathrm{~ms}$ whereas under native conditions, response delays up to $100 \mathrm{~ms}$ were observed. Fig $5 \mathrm{~F}$ depicts a major population shift to shorter response times in another network.

Blocking of inhibitory GABAA receptors with bicuculline did not have a universal effect on network delay distributions. In networks 1-5 (Table 1) there were a combined total of $33 \mathrm{MBLs}$ during native activity. Of these, only 14 remained leaders after exposure to bicuculline. A comparison of these 14 network delay distributions in the native and bicuculline state revealed that peak delays of five shifted to the right, four shifted to the left and five showed no obvious change (Table 2). The distribution widths at half max for six network delay distributions expanded (average percent difference : 9 ), while seven compressed (average percent difference : 28 ). One showed no change. The changes in this parameter are network specific. Networks 2, 3, and 5 compress, but networks 1 and 4 expand. However, the predominant effect appears to be a tightening of the distribution width.

\begin{tabular}{llllllll} 
N\# & MBL\# PDS & \%dhm & \%dpa & LPbb LPab \%dLP \\
\hline 1 & 8 & 0 & 0 & 6 & 8 & 20 & 43 \\
1 & 32 & 0 & 5 & 1 & 9 & 32 & 56 \\
1 & 56 & 9 & 9 & -4 & 12 & 12 & 0 \\
2 & 6 & 4 & -28 & 31 & 9 & 14 & 22 \\
2 & 27 & 0 & -16 & 18 & 6 & 10 & 25 \\
2 & 29 & 0 & -17 & 19 & 9 & 9 & 0 \\
2 & 32 & 0 & -16 & 19 & 13 & 7 & -30 \\
2 & 33 & -3 & -28 & 26 & 13 & 10 & -13 \\
3 & 7 & -6 & -61 & 61 & 14 & 22 & 22 \\
4 & 3 & 14 & 8 & -10 & 13 & 21 & 24 \\
4 & 8 & 11 & 21 & -17 & 32 & 12 & -46 \\
4 & 20 & 2 & 4 & -15 & 18 & 21 & 8 \\
4 & 24 & -4 & 8 & 6 & 4 & 11 & 47 \\
5 & 4 & -16 & -35 & 25 & 42 & 23 & -29 \\
\hline Means & Neg: -7.3 & Exp 7.9 & Inc: 21.2 & 14.4 & 16 & Inc: 30.7 \\
& & Pos: +4 & Cmp: 28.7 & Dec: 12 & & Dec: 29.4
\end{tabular}

TABLE II: N\#: Network Number; MBL\#: Major burst leader number; PDS: Peak delay shift $(\mathrm{ms}) ; \% \mathrm{dhm}$ : \% Diff in width at 1/2 max; \%dpa: \% Diff in peak amplitude; LPbb: Burst leadership percent before bicuculline; LPab: Burst leadership percent after bicuculline; \%dLP: \% Diff in burst leadership potential, Neg mean decrease, Pos mean increase, Exp Expansion of distribution (broadening), Cmp Compression of distribution (narrowing), Dec mean amplitude decrease (negative), Inc mean amplitude increase (positive), Inc mean \% increase in LP, Dec: mean decrease in burst leadership potential

\section{Paired Response Correlation and Peak Delays}

Followers do not participate in every network burst event (Fig 2) and their RDDs are a function of the specific burst leader. To examine how often followers responded to MBLs we used a paired response correlation (PRC; Methods) scheme that ranged up to $100 \%$ and was unique for leader follower pairs. Distributions with PRCs less than $5 \%$ are in the noise level and such distributions were not used in any analysis dependent on PRCs. In Fig 6A we show RDDs, in $2 \mathrm{~ms}$ bins, generated by three MBLs acting individually on one follower with $\mathrm{PRC}$ values of 85,62 and $44 \%$.

In each of the 33 native medium MBLs in networks 1-5, average PRC values decreased as peak delays increased. Best logarithmic trend line fit (mean R2 $=0.78 \quad 0.2$ ) occurred when followers of each MBL were grouped by peak delay into 5 or $10 \mathrm{~ms}$ bins and PRCs were averaged (Fig $6 \mathrm{~B})$. Such observations show that long peak delay times are associated with a lower probability of responding to a leader. It should be noted that PRCs were a function of the burst leader (6B). Since long peak delays in these small networks most likely involve inhibitory synapses, this offers an explanation as to why removal of inhibition tends to increase PRCs, (Fig 5A-D) shorten peak de- 
lays (Fig 5B) and narrow distributions (Figs 5E-F). High PRCs combined with low MRDs and short peak delays indicate strong coupling between a leader/follower pair.

\section{Distance and response delay distributions}

In many cases, a follower neurons physical distance from the burst leader appears to be a factor in the generation of its RDD. In 28 out of 33 MBLs in networks 1-5, large follower peak delays (10 ms bins) correlated to large average distances in the recording matrix. Fig 7A shows follower locations and RDDs for a single MBL (location circled). Most rapid responses occurred near the burst leader. It is important to stress that only the average distance increased with peak delay therefore exceptions could be found. For example, an arrow points to a neuron relatively far from the burst leader $(750 \mathrm{~m})$, which had a short peak delay. Fig 7B shows three examples of the relationship between peak delay in $10 \mathrm{~ms}$ bins and average distance. A similar relationship was seen when minimum response delays were plotted against average distance. Fig $7 \mathrm{C}$ plots the MRDs from the data of $7 \mathrm{~A}$ in $2 \mathrm{~ms}$ bins against the average distance per bin. It is likely that with increasing MRD and increasing distance, a larger number of serial synaptic steps are involved.

\section{Network Connectivity}

Morphological network circuitry is difficult to establish because many connections are made between neurons, even in widely distributed low density networks. This requires that emphasis be placed on functional connectivity to determine neuronal circuit structures. We hypothesize that the smallest delay between a leader follower pair, the $\mathrm{MRD}$, can be used to approximate the minimum number of synaptic connections existing between the pair. As indicated previously in Methods, a delay of $2 \mathrm{~ms}$ correlates to a mono-synaptic connection. Similarly, a follower with a $4 \mathrm{~ms}$ response delay can be thought of as being 2 synapses away from the burst leader.

In Fig 8, we show followers grouped by their minimum response delays (in 2ms bins) to burst leaders 6 and 32 from the same network. Signals originating from different burst leaders did not activate followers in the same order. For example: when neuron 32 leads $(8 \mathrm{~A})$, neuron 11 (arrow) is three bins away from neuron 5 (arrow). However, when neuron 6 leads $(8 \mathrm{~B})$, they share the same bin. It should be noted that the first bin generally contained about half of the followers, and all other major burst leaders (grey squares). Neurons in this bin are thought to be in contact with the MBL through at least one single synapse connection. Hence, in the small networks analyzed, the majority of neurons have mono-synaptic connections to the major burst leaders.
Functional connectivity is also inferred using Paired Response Correlation (PRC) values. In Fig 9, connectivity in network 1 is examined using three different PRC thresholds (50, 70 and 90\%). Directed graphs with edges connecting burst leaders (filled squares) to followers (white squares) meeting or exceeding the threshold were made. Dots represent followers with PRCs less than $50 \%$. Fig 9 shows results from network 1 during hours 10-15 (Fig 3C). At 50\% (9A), eleven neurons connected to each MBL. The majority of these highly connected neurons were also major burst leaders $(8,23,32,40$, 45,56 , and 61). Altogether, nineteen out of 62 neurons responded (within 100ms) to at least one major burst leader $50 \%$ of the time or higher. Each follower neuron connected to an average of 5.12.1 MBLs. With a threshold of $70 \%$ (9B), six neurons, 23, 32, 40, 45, 56, and 61 (all MBLs), connected to each MBL. Twelve neurons remained connected at this level and each contacted an average of 4.52.0 MBLs. At a threshold of 90\% (9C), one neuron (45) connected to all MBLs. A total of 8 neurons remained connected at this level, and each contacted an average of 2.81.8 MBLs.
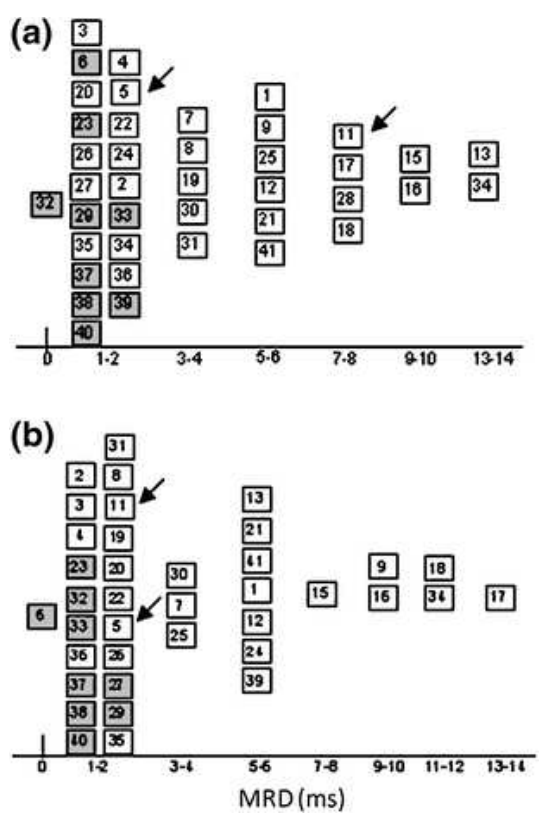

FIG. 8: Minimum response delays can be used to determine network connectivity. (A) MRD pattern to burst leader 32 . (B) MRD pattern to burst leader 6. Both are from the same network. Shaded boxes are major burst leaders. Burst leaders do not activate followers in the same order. Arrows show two followers with MRDs that vary by burst leader. The first two columns represent the same MRD time period.

Fig 9C reveals a highly connected circuit of neurons. This circuit contained all of the major burst leaders, which, for this particular network, were responsible for initiating $75 \%$ of all network bursts. High connectivity between MBLs indicates that even if an MBL did not 
start a network burst, it was very likely to participate in that network burst and, presumably, help propagate the burst by recruiting closely coupled follower neurons. Similar findings were found in all study networks. RDDs where one MBL followed another MBL showed high levels of connectitvity with MRDs around $2 \mathrm{~ms}$ and PRCs above $70 \%$. Therefore, we conclude that this 'primary circuit,' composed mainly of MBLs, is responsible for the continual ignition of the network and generation of longterm spontaneous activity.

\section{DISCUSSION}

In this manuscript, we show that major burst leaders are spontaneously active cells that play an important role in triggering network burst events. Such cells typically display higher levels of spike activity and form a monosynaptically connected primary circuit. Together, burst leaders dominate the initiation of spontaneous network firing patterns via recruitment of other cells or cell clusters. Here, we explored three features of the activation mechanisms underpinning network bursts. First, major burst leaders (MBLs), which comprise a minority of cells in the network, form well-connected circuits with each other, and collectively lead most network bursts. Followers exhibit response delay distributions, paired response correlations, and minimum response delays unique to individual MBLs (Figs 4A, 8) suggesting that different MBLs will uniquely influence network information flow, information storage, its retrieval and responses to incoming sensory stimuli without modification to existing synaptic infrastructure. Second, detailed temporal lag relationships between $\mathrm{MBL} /$ follower pairs were characterized with response delay distributions (RDDs), which established that responsiveness is a decreasing function of physical distance and number of synaptic connections between cells. RDDs have three important features: (1) minimum response delays (MRDs), which provide information about the minimum number of synaptic connections that exist between a leader/follower pair; (2) the paired response correlation (PRC), which represents a weighting factor for the connection between a leader/follower pair (see Fig 9 where PRCs were used to graph functional connectivity); and (3) the peak delay, which represents the delay time corresponding to the highest probability of follower response. Third, we showed that blocking GABAA inhibition narrows response delay distributions and results in drastic changes to the burst leader pool. The abruptness with which network activity changes after the addition of bicuculline suggests that existing network circuitry, along with inherent conduction and synaptic delays remain unchanged while only computational (processing) activities are modified.

In approximately $18 \%$ of all cases, individual neurons increased their minimum response delay following the addition of $40 \mathrm{M}$ bicuculline (Fig 5C), a common concentration for pharmacological studies. This counterintuitive result might be explained by noting that $40 \mathrm{M}$ is not a saturating concentration. Therefore, some inhibitory circuitry could remain active and have greater inhibitory influence associated with increased firing rates. By acting on key connections, greater inhibition could increase minimum response delays or even expand network delay distributions (Table 2). While, the primary effect remains a sharpening of the phase delay distributions, the effects of bicuculline concentration levels on population response dynamics warrants further study. An issue common to all network wide measurements of neural activity, even in relatively small networks in vitro, is that present recording methods account only for a fraction of the neuronal activity. Recent research indicates that voltage sensitive dyes and optical recording methods are coming very close to revealing the activity of each individual network neuron $[12,13]$, but at this time electrophysiological recordings on microelectrode arrays (MEAs) still provide the best access to multiple individual neuronal signals with fine temporal resolution. Nevertheless, we estimate that data obtained from MEA recordings typically represents only about $5-10 \%$ of total neurons present and may be biased towards more active neurons and those that somehow project stronger signals. Therefore, the first neuron detected in a network burst could represent either a true ignition site or the fastest recorded follower of an ignition site outside the recording window. Despite this limitation, the identification of major burst leaders (MBLs) demonstrates that specific ignition sites are involved in generating spontaneous network activity.

Mechanisms similar to those creating strong basic driving forces in spontaneously active self-organized small networks in vitro are likely to be found in natural central nervous system activity. We hope that these quantitative characterizations of general collective spontaneous activity patterns in vitro will serve to guide and constrain future models and lead to greater understanding of the computational properties of nervous systems.

\section{ACKNOWLEDGEMENTS}

This project supported by LDRD-ER-20050411, and in part by the Texas Advanced Technology Program

[1] A. Artola and W. Singer. NMDA receptors and developmental plasticity in visual neocortex. In: The NMDA receptor. London: Oxford UP, 1994.

[2] J. M. Beggs and D. Plenz. Neuronal avalanches are diverse and precise activity patterns that are stable for 

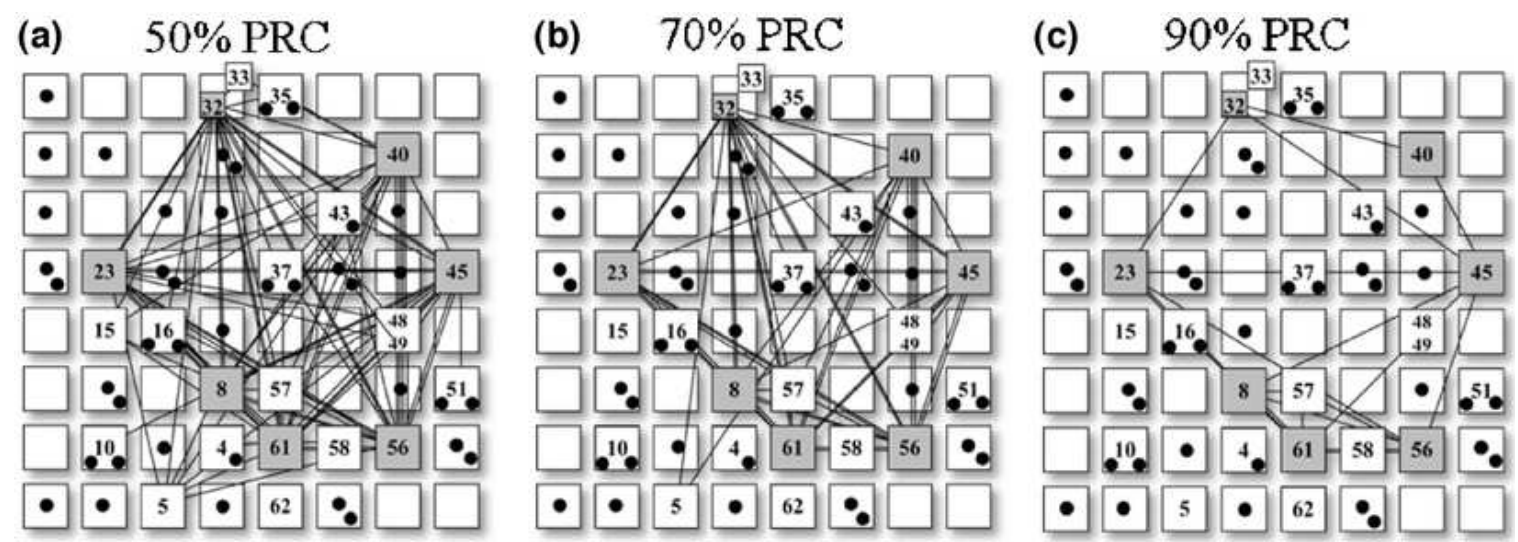

FIG. 9: Pair-wise response correlations (PRCs) used to determine network connectivity. Burst leaders are shown with edges connecting to followers that exceed a PRC threshold of (A) $50 \%$ (B) $70 \%$ and (C) $90 \%$. Filled squares are burst leaders; boxes with numbers are followers with at least one PRC value greater than 50\%; dots are followers with PRCs less than $50 \%$.

many hours in cortical slice cultures. J. Neurosci., 24:5216, 2004.

[3] Y. Ben-Ari. Developing networks play a similar melody. Trends Neurosci, 24:353-60, 2001.

[4] Luis M. A. Bettencourt, Greg J. Stephens, Michael I. Ham, and Guenter W. Gross. Functional structure of cortical neuronal networks grown in vitro. Phys. Rev. E, 75(2):021915, 2007.

[5] T.V. Bliss and G.L.A Collingridge. A synaptic model of memory: long-term potentiation in the hippocampus. Nature, 361:31-39, 1993.

[6] M.A. Corner, J. Van Pelt, P.S. Wolters, R.E. Baker, and R.H. Nuytinck. Physiological effects of sustained blockade of excitatory synaptic transmission on spontaneously active developing neuronal networks?an inquiry into the reciprocal linkage between intrinsic biorhythms and neuroplasticity in early ontogeny. Neurosci Biobehav Rev, 26:127-185, 2002.

[7] D.R. Curtis, A.W. Duggan, D. Felix, and F.A.R. Johnston. Gaba, bicuculline and central inhibition. Nature, 226:1222-1224, 1970.

[8] P. Darbon, L. Scicluna, A. Tscherter, and J. Streit. Mechanisms controlling bursting activity induced by disinhibition in spinal cord networks. Eur J Neurosci, 15:671-683, 2002.

[9] T. B. DeMarse, D. A. Wagenaar, A. W. Blau, and S. M. Potter. The neurally controlled animat: Biological brains acting with simulated bodies. Auton. Rob., 11:305, 2001.

[10] M.H. Droge, G.W. Gross, M.H. Hightower, and L.E. Czisny. Multielectrode analysis of coordinated, multisite, rhythmic bursting in cultured cns monolayer networks. $J$ Neurosci, 6:1583-1592, 1986.

[11] D. Eytan and S. Marom. Dynamics and effective topology underlying synchronization in networks of cortical neurons. J Neurosci, 26:8465-8476, 2006.

[12] O. Feinerman, M. Segal, and E. Moses. Identification and dynamics of spontaneous burst initiation zones in unidimensional neuronal cultures. J Neurophysiol, 97(4):29372948, 2007.

[13] A. Grinvald and R. Hildesheim. Vsdi: a new era in functional imaging of cortical dynamics. Nat Rev Neurosci, $5: 874-885,2004$.
[14] G. W. Gross and F. U. Schwalm. A closed flow chamber for long-term multichannel recording and optical monitoring. J. Neurosci. Methods, 52:73, 1994.

[15] G.W. Gross. Internal dynamics of randomized mammalian neuronal networks in culture. In: Enabling technologies for cultured neural networks. New York: Academic, 1994.

[16] G.W. Gross and J.M. Kowalski. Origins of activity patterns in self-organizing neuronal networks in vitro. J Intel Mater Syst Struct, 10:558-564, 1999.

[17] G.W. Gross, W.Y. Wen, and J.W. Lin. Transparent indium-tin oxide electrode patterns for extracellular, multisite recording in neuronal cultures. Neurosci Methods, 15(3):243-252, 1985.

[18] Y. Jimbo, H.P.C. Robinson, and A. Kawana. Strengthening of synchronized activity by tetanic stimulation in cortical cultures: application of planar electrode arrays. IEEE Trans Biomed Eng, 45:1297-1304, 1998.

[19] Y. Jimbo, T. Tateno, and H.P.C. Robinson. Simultaneous induction of pathway-specific potentiation and depression in networks of cortical neurons. Biophys $J$, 76:670, 1999.

[20] H. Kamoika, E. Maeda, Y. Jimbo, H. P. C. Robinson, and A. Kawana. Spontaneous periodic synchronized bursting during formation of mature patterns of connections in cortical cultures. Neurosci Lett, 206:109, 1996.

[21] L.C. Katz and C.J. Shatz. Synaptic activity and the construction of cortical circuits. Science, 274:1133-1138, 1996.

[22] E.W. Keefer, A. Gramowski, and G. W. Gross. Nmda receptor dependent periodic oscillations in cultured spinal cord networks. J. Neurophysiol., 86:3030, 2001.

[23] X. Leinenkugel, R. Khazipov, R.C. Cannon, H. Hirase, Y. Ben Ari, and G. Buzsaki. Correlated bursts of activity in the neonatal hippocampus in vivo. Science, 296:20492052, 2002.

[24] E. Maeda, H. P. C. Robinson, and A. Kawana. The mechanisms of generation and propogation of synchronized bursting in developing networks of cortical neurons. $J$. Neurosci, 15:939, 1995.

[25] S. Marom and G. Shahaf. Development, learning and memory in large random networks of cortical neurons: 
lessons beyond anatomy. Q. Rev. Biophys., 35:63, 2002.

[26] M. Meister, R.O.L. Wong, D.A. Baylor, and C.J. Shatz. Synchronous bursts of action potentials in ganglion cells of the developing mammalian retina. Science, 252:939943, 1991.

[27] K. Nakanishi and F. Kukita. Functional synapses in synchronized bursting of neocortical neurons in culture. Brain Res, 795:137-146, 1998.

[28] J. Van Pelt, P.S. Wolters, M.A. Corner, W.L.C. Rutten, and G.J.A Ramakers. Long-term characterization of firing dynamics of spontaneous bursts in cultured neural networks. IEEE Trans BioMed Eng, 51(11):2051-2062, 2004.

[29] M. Puopolo and O. Belluzzi. Nmda-dependent, networkdriven oscillatory activity induced by bicuculline or removal of $\mathrm{mg} 2+$ in rat olfactory bulb neurons. Eur $J$ Neurosci, 13:92-102, 2001.

[30] G.J.A. Ramakers, H. Van Galen, M.G.P. Feenstra, M.A. Corner, and G.J. Boer. Activity-dependent plasticity of inhibitory and excitatory amino acid transmitter systems in cultured rat cerebral cortex. Int J Devl Neurosci, 102:611-621, 1994.

[31] R.R.Provine. Wing-flapping? develops in wingless chicks.
Behav Neur Biol, 27:233-237, 1979.

[32] J.V. Selinger, J.J. Pancrazio, and G.W. Gross. Measuring synchronization in neuronal networks for biosensor applications. Biosens and Bioelectron, 19(7):675-683, 2004.

[33] G. Shahaf and S. Marom. Learning in networks of cortical neurons. J Neurosci, 21:8782-8788, 2001.

[34] N.C. Spitzer. Electrical activity in early neuronal development. Nature, 444:707-712, 2006.

[35] Y. Takikawa, R. Kawagoe, and O. Hikosaka. Rewarddependent spatial selectivity of anticipatory activity in monkey caudate neurons. J Neurophysiol, 87:508-515, 2002.

[36] D.A. Wagenaar, J. Pine, and S.M. Potter. An extremely rich repertoire of bursting patterns during the development of cortical cultures. BMC Neurosci, 7:11, 2006.

[37] D.A. Wagenaar, M. Radhika, J. Pine, and S.M. Potter. Controlling bursting in cortical cultures with closedloop multi-electrode stimulation. J Neurosci, 25:680-688, 2005.

[38] L.I. Zhang and M.M. Poo. Electrical activity and development of neural circuits. Nat Neurosci, 4:S1207-S1214, 2001. 